pointed out that such a syphilitic rash is due, not to syphilis introduced at the time of vaccination, but to a congenital infection. No rash in the case of inoculated syphilis ever appears before, at the very earliest, ffty days after inoculation. Apparently in the case of a child suffering from congenital syphilis, vaccinia produces enough local irritation and general constitutional disturbance to cause the appearance of skin manifestations, vaccination simply determining the date of the appearance of the skin lesions. Notwithstanding the fact that calves are absolutely immune against syphilis, congenital syphilitic rashes much more frequently appear after vaccination with calf than with humanised lymph.

Group II. consists of post-vaccinal rashes more or less preventable. Certainly Section $A$, those due to a virus plus the vaccine virus introduced at the operation of vaccination, are, with the exercise of ordinary precautions, absolutely preventable, and when occurring indicate culpable negligence on the part of the operator.

Subsection (a).-As a result of the introduction of pus cocci impetigo contagiosa may occur. This is a very rare accident, accidental inoculation taking place much more frequently after rupture of the vaccine vesicles. Short of true impetigo contagiosa slight forms of superficial inflammation may occur as a result of the introduction of one or other of the organisms found in pus.

Subsection (b). - The occasional inoculation of syphilis with vaccinia cannot be denied and is the great weapon in the hands of the anti-vaccinationists, but it has happened so rarely as practically to become a clinical curiosity, and with the introduction into general use of calf lymph will disappear altogether. When syphilis is inoculated simultaneously with vaccine virus, according to Fournier three alternatives present themselves: (1) syphilis is not transmitted but vaccinia is; (2) syphilis is transmitted and vaccinia fails; and (3) both are transmitted, some points of inoculation giving rise to typical vaccine vesicles, whilst later a syphilitic chancre or chancres appear at a point of inoculation when vaccinia has failed. Hutchinson states that a syphilitic chancre may follow typical vaccine vesicles in the same site. Fournier gives an excellent table in his clinical lecture on the subject, as to the differential diagnosis of vaccinosyphilis which is too long to give here. Briefly, he points out that the syphilitic chancre is limited to one or two points of inoculation, inflammation is slight, loss of substance is superficial, and the parchment induration is typical, not inflammatory. The acquired syphilitic rash appears at the earliest from fifty to ninety days after vaccination and requires in every case the existence of a specific sore at the site of the vaccination. In fact, vaccino-syphilis is in no way different from syphilis acquired in other ways and follows precisely the same course.

In this country the question of the introduction of leprosy with vaccinia is purely academic and the reported evidence from other countries is very conflicting. Tuberculosis is a disease which is confidently asserted by the anti-vaccinationists as likely to be introduced by vaccination. In Germany many experiments have been made with lymph from tuberculous patients with uniformly negative results. Attempts to inoculate the skin of guinea-pigs-animals most susceptible to tuberculosis-have invariably failed. That the skin of the human organism, however, is susceptible to the inoculation of tubercle cannot be denied, as cases have been reported where a local tuberculosis has been produced from tattooing and the like. Recently at the Dermatological Society of London a case was shown in which, although there was no microscopical or bacteriological evidence, yet the majority of those present agreed that the appearance was that of a local tuberculosis the result of vaccination. The question, however, of the transmission of the three diseases, syphilis, leprosy, and tuberculosis, by vaccination is probably solved by the introduction of glycerinated calf lymph by the new Vaccination Act of the present Government and ought to go a long way towards disarming what might be a legitimate criticism of vaccination.

Section $B$ of Group II. comprises rashes due to the introduction of a virus after the formation and rupture of the vaccine vesicles. Of these by far the most important is erysipelas. It is possible that the pathogenic organism of erysipelas may be introduced into the patient on the eighth day by the operator opening the vesicles, but the accident must be excessively uncommon. After the opening or rupture of the vesicles the introduction of the erysipelas organism is not so uncommon and in the large majority of cases occurs when the child is living in bad hygienic surroundings. The characteristic rash of erysipelas generally appears within 24 hours after inoculation around the vesicles and spreads rapidly. In young infants it is very fatal and it was on this account that in the new Vaccination Act it was first proposed to raise the age limit at which vaccination must have been performed from three months (as now) to one year, statistics showing that the death-rate from erysipelas gradually diminishes with each succeeding month of the first year of life. Cellulitis mentioned in the classi. fication is probably erysipelas complicated with pus cocci. Impetigo contagiosa is not uncommon after the rupture of the vesicles, around which it spreads and by the child's fingers may be carried to other parts of the body. Furunculosis is described as occurring as the result of the absorption of pus cocci into the system, their dissemination and ultimate arrest in the skin.

Gangrene local or disseminate. A local gangrenous action the result of septic poisoning may sometimes be seen at the site of the vaccination. A large black slough forms, which when detached leaves a deep ulcer which takes weeks to heal. Clement Lucas has reported a fatal case in an infant three months old. Disseminated gangrene the result of vaccinia is an exactly similar condition to that described as varicella gangrenosa. The lesions commence as a pin's-bead sized papulo-pustule generally on the vaccinated arm and appear all over the body in crops. 'The pustule ruptures. a scab forms with an inflammatory aerola, and the centre becomes a black slough. Barlow has pointed out that the majority of cases, whether occurring after varicella or vaccinia, are associated with tuberculosis.

Cases of pyæmia after vaccination have been reported. As clinical curiosities may be mentioned the case published by Diday where a growth of hair took place around the cicatrix of a vaccination performed on the anterior part of the thigh of an infant eleven months old, and the occasional occurrence of keloid in the vaccination cicatrix, two cases of which have been reported.

Acacia-road, N.W.

\section{NOTES ON THE}

\section{LOCALISATION OF A CENTRE IN THE BRAIN CORTEX FOR RAISING THE UPPER EYELTD.}

\section{Bx W. HARTLEY BUNTING, M.D., F.R.C.S. EDIN.}

Although most of the motor centres in the brain cortex have for some time been accurately defined the centre for raising the upper eyelid is as yet uncertain. The very existence of such a centre has indeed been doubted by some, who hold that the centre for the levator palpebræ superioris along with that for all the extrinsic muscles of the eyeball is intracortical and is situated somewhere in the region of the corpora quadrigemina. I think, however, that there is strong evidence to show that a cortical centre does exist and that it is situated far forward in the general motor area. There are some difficulties which present themselves in the way of experimentally localising this centre. In the first place, since the eyelids on the two sides under normal conditions act together, one finds that the centres in the two halves of the brain for raising the lid are so intimately associated that compensation after destruction of the centre on one side readily takes place and ptosis is frequently not observed at all, or, if noted, it is quite temporary, the opposite side taking on the function; this theory was first advanced by Wernicke. Horsley as the result of the study of a clinical case arrived at the same conclusion, and he says that when cortical ptosis exists from damage to one side only of the brain cortex the centres for the elevators of the upper lid are not so closely associated as usual. Again, fallacies have doubtless arisen from the fact that all the external ocular muscles, the levator palpebræ included, may be excited to coördinated movements by vigorous stimulation in many parts of the brain cortex; moreover, it is but rarely that one has the opportunity of making a necropsy in a case of cortical ptosis, or if such an opportunity does occur, the lesion is probably so extensive as to render the results in this direction negative.

Landouzy and Grasset advanced the theory that a centre for movement of the upper lid could be localised in the angular gyrus. The lesions, however, in Landouzy's case 
were. I think, too extensive to admit of the definite conclusions he draws. Lemoine also quotes a case of longstanding ptosis in which, at the post-mortem examination, he found a lesion in the angular gyrus, and he agreed with Landouzy as to the position of the centre. Hirt and others, more recent writers, also hold this opinion. Ferrier thinks that these conclusions are not warranted by experiment. $\mathrm{He}$ says, "Some such cases" (that is, of ptosis due to a small lesion in a definite area of the cortex) " have been observed in connexion with diseases of the cortex, and attempts have been made, but not, I think, successfully or in accordance with experimental lesions, to localise this centre in the angular gyrus. If a distinct volitional centre for the levator palpebræ superioris exists in the human brain I think it must be sought for at the base of the first frontal and extending partly into the second frontal convolution of the brain. Here is an area, irritation of which in the brain of the monker causes elevation of the eyelids." Ferrier found, moreover, in applying stimulation to this area that if the stimulus were very faint elevation of the lids was the only movement that followed. Although movement of the eyelids can be seen on stimulating the angular gyrus the latent period, as pointed out by the same observer, is onehundredth of a second longer than in the case of stimulation applied to the oculo-motor centre in the frontal lobe. Schüfer and Ferrier both found that when the angular gyrus was destroyed on both sides in the brain of a monkey, no ptosis was produced; and movements of the lids caused by stimulating this area are probably due to association with the frontal region. In one of Ferrier's experiments he extirpated the greater part of the frontal lobe in a monkey ; ptosis with some disturbance of ocular movement was the only paralytic effect produced.

De Bono, as a result of the study of clinical cases, came to the conclusion that a cortical centre for the levator palpebræ superioris could be localised in the fore part of the brain. The two cases from which he chiefly drew his conclusions were both in young women affected with ptosis and subject to epileptiform attacks. In the one case the ptosis was congenital, and in the other it was of four years' duration. The epileptiform attacks in both cases began in the arms, and spread sometimes to the face and legs. De Bono thought that the lesion which caused the ptosis was also very probably the focus from which started the convulsions; and upon this he founded his theory that the centre for elevation of the lid must be closely associated with the centre for movement of the arm. Examination of the skull of the patient suffering from congenital ptosis revealed a depression about one finger's width in the zygomo-Rolandic line, $93 \mathrm{~mm}$. from the external auditory meatus and $102 \mathrm{~mm}$. from the orbital margin. This appeared to have resulted from an injury at birth, which was a difficult one, and it was considered by De Bono to be the cause of the ptosis. A study of similar heads showed that the depression corresponded to the anterior central (or ascending frontal) convolution at the junction of the middle with the upper two-fifths. Experiments on living dogs seemed to confirm this observation; and De Bono arrived at the conclusion that a centre for elevation of the lid of the opposite side could be localised in the anterior central convolution rather above the middle in front of the motor centre for the arm and above that for the face. These results of clinical observation are very similar to those arrived at by Ferrier's experiments.

Several cases of ptosis, as a result of blows upon the top of the head, have been reported. Thus Mr. Hulke, in the Reports of the Royal London Ophthalmic Hospital, gives such a case, which he thought was due to direct injury to the cortical centre, but it is more probable that such results are, generally at least, due to a lesion at the base. The following case, however, which is from the notes of a case which was under my own observation, points strongly, I think, to frontal localisation of the cortical centre for the levator of the eyelid.

A boy, aged twelve years, was swinging round a giant's stride when the iron wheel and arms on the top of the pole came off and fell, striking him on the top of the head above the right eye. When I first saw him, sixteen hours later, he was semi-conscious and lay quietly with both eyes closed. unless irritated. He could, however, open both eyes widely and there was no paralysis of any muscle or group of muscles. On removal of the carbolic compress which had been applied a large spoonful of brain matter was seen protruding from the anterior extremity of a large jagged wound situated in the right frontal region. The wound extended about three inches back from the brow and was about one and a half inches in width, the middle line of the head being about one and a half inches from the upper edge of the wound. Beneath this the skull was crushed in like the broken shell of an egg, making a depression in which two fingers could lie. On giving chloroform and enlarging the wound the fracture in the outer table of bone was seen to reach to the coronal suture, and the fracture of the inner table about half an inch to one inch beyond. The membranes were extensively torn, and the depressed part of the skull was driven into the brain especially in front. Owing to the extensive fracture of the inner table of the skull I had some difficulty in elevating, having to remove three circles of bone with the trephine. About two or three drachms of brain matter, over and above what had escaped before, were lost during the operation chiefly from the posterior end of the wound. The bone removed by the trephine was replaced and the wound was dressed. The patient remained in a semi-conscious condition for twelve days, and then steadily recovered. There was never any sign of meningitis, and no paralysis occurred with the exception of the left eyelid. Here ptosis appeared directly after the operation. and persisted for ten weeks. It was never absolute and gradually passed off. At the end of twelve weeks it had practically disappeared. I saw the boy occasionally during the next five months. The ptosis entirely disappeared, and so far as could be seen there were no permanent evil results from the extensive head injury from which he had suffered.

Noting the position of the fracture and its relation to the coronal suture it is seen that the posterior end of the fracture, from which the brain matter chiefly escaped during the operation, just about reaches the ascending frontal convolution, and that the level is that of the second (or middle) frontal convolution and the sulcus between the first and second convolutions. I believe that in this case the monolateral ptosis was the result of destruction of the cortical centre, probably during the elevation of the depressed bone, and that recovery was due to the function being assumed by the opposite side. I think that one may exclude the possibility of the ptosis being a nuclear paralysis because there was no ptosis to be observed sixteen hours after the accident and before the operation, but immediately after the operation ptosis was observed, which, however, did not increase in severity.

After weighing the evidence I think that the following conclusions are warranted:-1. There is a distinct cortical centre for the elevator of the upper eyelid. 2. This centre is situated in the posterior extremity of the second frontal convolution, extending probably into the first frontal convolution; that is, above and in front of that suggested by De Bono, and rather lower than the position assigned by Ferrier. 3 . There is a definite relation between this centre and the levator palpebræ superioris of the opposite side, and destruction causes paralysis, temporary or permanent, of the lid on the opposite side. 4. This cortical centre is distinct from the cortical centre or centres for the other eye muscles; and therefore, as a result of a cortical lesion, ptosis may occur as a solitary paralysis; or on the other hand, in cortical paralysis of the muscles of the eye, the levator may alone escape. It is interesting to note that the blood-supply of this cortical area is double, it being supplied both by the anterior cerebral and the middle cerebral arteries, which may account for the rarity of ptosis from cerebral embolism. Edinburgh.

\section{FRACTURE OF THE PATELLA. BY HEATHER BIGG, F.R.C.S. EDIN.}

THE immediate result of transverse fracture of the patella is that the tibia is more or less completely severed from its connexion with the extensor muscles of the thigh and the natural object of all treatment must therefore be to reunite this connexion between the two parts with as much strength and completeness as possible so that the powers of the knee joint may be restored to their fullest ability in walking. In the present days o advanced surgery this end is frequently attained by the actual operation of suturing the fragments together. But this is not always admissible and the alternative method of apposing the segments by external 